Org Lett. 2016 June 03; 18(11): 2620-2623. doi:10.1021/acs.orglett.6b01047.

\title{
Synthesis of the Privileged 8-Arylmenthol Class by Radical Arylation of Isopulegol
}

\author{
Steven W. M. Crossley $\ddagger$, Ruben M. Martinez ${ }^{\ddagger}$, Sebastián Guevara-Zuluaga, and Ryan A. \\ Shenvi ${ }^{*}$ \\ Department of Chemistry, The Scripps Research Institute, 10550 N Torrey Pines Rd, La Jolla, CA \\ 92037, United States
}

\begin{abstract}
Hydrogen atom transfer (HAT) circumvents a disfavored Friedel-Crafts reaction in the derivatization of the inexpensive monoterpene isopulegol. A variety of readily-prepared aryl and heteroaryl sulfonates undergo a formal hydroarylation to form 8-arylmenthols, privileged scaffolds for asymmetric synthesis, as typified by 8-phenylmenthol. High stereoselectivity is observed in related systems. This use of HAT significantly extends the chiral pool from the inexpensive monoterpene isopulegol.
\end{abstract}

\section{Graphical abstract}

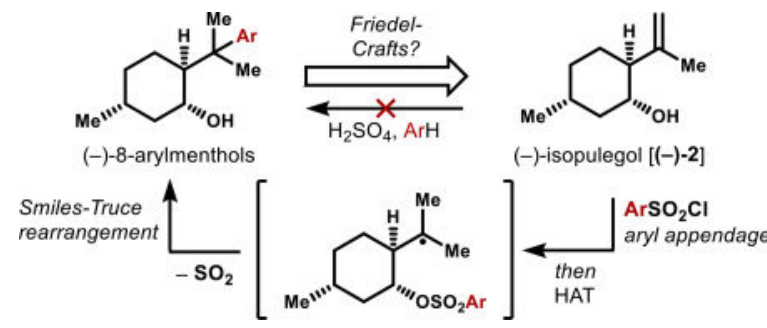

New chemical reactions to modify abundant terpenes find application in polymer science, medicine and chemistry, as well as the flavor and fragrance industry. ${ }^{1}$ Chiral catalysts, reagents and auxiliaries, for example, can be sourced from cyclic monoterpenes, ${ }^{2}$ which are usually inexpensive, abundant and easily removed from reaction mixtures. During recent studies of the isocyanoterpene (ICT) class, ${ }^{3}$ we required access to aryl derivatives of the broadly-applicable chiral controller, 8-phenylmenthol (1). ${ }^{4}$ The widely applied parent alcohol (-)-1 or (+)-1 can be appended to carboxylic acids to effect highly stereoselective reactions, as shown in Figure 1a,b. ${ }^{5,6}$ Many reports have also documented substantial variations in diastereocontrol achieved due to variations in the identity of the aryl group ${ }^{7,9 b, c, e-i}$ but short routes to these aryl analogs are not available.

Corresponding Author. rshenvi@scripps.edu.

¥These authors contributed equally.

Dedicated to E.J. Corey.

ASSOCIATED CONTENT

Supporting Information. Detailed experimental procedures, spectral data, chromatograms and x-ray crystallography. This material is available free of charge via the Internet at http://pubs.acs.org. 
Although either enantiomer of $\mathbf{1}$ is commercially available, the high cost [(-)-1: 340 \$/g; (+)-1: $840 \$ / \mathrm{g}$ ] and low supply of these molecules impedes direct functionalization, ${ }^{7}$ and probably reflects the cost of their 5 or 6-step syntheses from (+)- or (-)-pulegone (3.4 $\$ / \mathrm{g}$ at $97 \%$ purity and 206 \$g, respectively; prices from Sigma; see Figure 1c). ${ }^{4}$ Desymmetrization of ( \pm )-trans-2-cumyl-cyclohexanol using a lipase can be an effective alternative to $1,{ }^{8}$ but yields range from $31-48 \%$ over two resolution cycles and commercial costs are high (214 \$/g for (-)-2-cumylcyclohexanol; (+)-isomer discontinued). Analogs of 1 have been synthesized by Corey's route, ${ }^{9}$ but are not widely available, and we could find no examples in the literature of heteroaryl analogs. ${ }^{10}$

Here we report a radical method that provides rapid access to (+)- and (-)-8-phenylmenthol and numerous chiral arylated menthol derivatives. Combination of hydrogen atom transfer (HAT) radical generation ${ }^{11,12}$ with a radical Smiles-Truce rearrangement ${ }^{13,14}$ allows homoallylic alcohols to be formally hydroarylated using arylsulfonyl halides. Electrondeficient, -neutral and -rich arenes may be engaged, as well as electron-rich sites on heterocycles, orthogonal to the Minisci reaction.

The obvious precursor to (-)-8-phenylmenthol is (-)-isopulegol [(-)-2], whose low cost of $0.10 \$ / g$ reflects its intermediacy in the multi-kiloton industrial synthesis of (-)-menthol itself (Figure 2).${ }^{15}$ However, direct Brønsted acid-mediated Friedel-Crafts phenylation of isopulegol results in complex mixtures due to selective ionization of the alcohol function. Therefore, we turned away from a cationic mechanism to investigate radical arylation.

We previously reported that carbon-centered radicals generated under Mukaiyama-type conditions ${ }^{16,17}$ are sufficiently persistent to engage proximal arenes. ${ }^{11 f, 18,19}$ Frequently, the chemoselectivity of these conditions is orthogonal to Brønsted acid mediation. To effect aryl transfer to the isopulegol alkene, we imagined appending an arene to the proximal alcohol with a single-electron nucleofuge (Figure 2). ${ }^{14}$ However, benzoates (entries 1-4) or (phenyl)silyl ${ }^{20}$ ethers (entries 5-6) led only to hydrogenation products $\mathbf{B}$ or recovered starting materials $\mathbf{A}$, even if strong electron-withdrawing groups were appended. The sulfonate linker alone (3, entry 7$)$ was competent to produce aryl transfer product (-)-1, which is the targeted auxiliary. Radical Smiles-Truce rearrangements reported by Motherwell, Studer, Tada and Stephenson also utilize a sulfonate radical nucleofuge, ${ }^{13,14}$ which appears to provide both Thorpe-Ingold acceleration and an inductive electron withdrawing effect. A range of metal complexes could affect this transformation in absence of $\mathrm{O}_{2}$, but stoichiometric metal reagents were required consistently, presumably due to metal sequestration via irreversible binding of sulfite radical $\left(\mathrm{ROSO}_{2} \bullet\right)$ or liberated sulfur dioxide $\left(\mathrm{SO}_{2}\right) \cdot{ }^{21}$ Despite extensive efforts, attempts to effect metal-turnover were unsuccessful, although addition of TBHP accelerated the reaction. ${ }^{22}$

As shown in Figure 3, we found that a diverse set of aryl sulfonates function well in this chemistry. Most of the arylsulfonyl chloride/fluoride precursors are commercially available and all are accessible in short sequences (see Supporting Information [SI]). Notably, anisole can be appended at its more electron rich ortho- (4a) and para- (4b) positions. As expected, electron-rich tolyl groups $(\mathbf{5 a - c})$ do not engage the electron-rich tert-alkyl radical as efficiently as electron-deficient halogenated aromatics $(\mathbf{6 a - c})$, but still competently generate 
the arylated products, which are easy to separate from hydrogenation byproducts. Naphthyl groups transfer efficiently (7-8), and the 1-naphthyl derivative (8) shows restricted bond rotation (see SI), illustrating the steric clash this rearrangement will tolerate. Even a 1pyrenyl substituent can be transferred: $\mathbf{9}$ may be of some utility for enantioselective catalysis. ${ }^{23}$ Pyridyl groups are very efficient in accepting alkyl radicals, as observed by Minisci in intermolecular settings, ${ }^{24}$ and we accordingly observed efficient 2-pyridyl transfer (10). However, in this case, electron-poor (11) or -rich (12) 3-pyridyl groups can be selectively engaged, whereas the 3-position is generally unreactive in bimolecular Minisci coupling. These nitrogen heterocycles appended to the menthyl skeleton are appealing chiral Lewis basis to investigate for enantioselective catalysis. Other heteroaromatics-quinolines, furans, thiophenes, thiazoles and benzothiazoles (13-17) all transfer efficiently. The benzofurazan will transfer (18), albeit in very modest yield. In this case, we attempted to improve the yield by varying the metal complex, but found instead that $\mathrm{Fe}(\mathrm{acac})_{3}$ caused double $\mathrm{N}-\mathrm{O}$ bond cleavage, ${ }^{25}$ and only trace aryl transfer product was observed.

Although use of stoichiometric $\mathrm{Mn}(\mathrm{dpm})_{3}$ is not cost-prohibitive, we discovered that its partial replacement with the inexpensive salt $\mathrm{Mn}(\mathrm{OAc})_{3} \cdot 2 \mathrm{H}_{2} \mathrm{O}$ could effectively lower its loading to $5 \mathrm{~mol} \%$, albeit with longer reaction times. The presence of $\mathrm{Mn}(\mathrm{OAc})_{3} \cdot 2 \mathrm{H}_{2} \mathrm{O}$ in solution presumably allows active $\mathrm{Mn}(\mathrm{dpm})_{\mathrm{n}}$ complex to reform in situ. Indeed, addition of $20 \mathrm{~mol} \%$ of ligand (Hdpm) with 1 equiv of $\mathrm{Mn}(\mathrm{OAc})_{3} \cdot 2 \mathrm{H}_{2} \mathrm{O}$ behaves identically to $5 \mathrm{~mol}$ $\% \mathrm{Mn}(\mathrm{dpm})_{3}$. At $1 / 20^{\text {th }}$ the price ( $\$ 1.1 \mathrm{vs} \$ 24 / \mathrm{mmol}$, Sigma) and much reduced formula weight, we found this to be convenient and scalable.

These optimized conditions were successfully applied to the scaled synthesis of both isomers of $\mathbf{1}$ (Figure 4), with (+)-1 originating from (-)-citronellal; (+)-isopulegol is more expensive than (-)-2. Cyclization of $\mathbf{1 9}$ occurred in high yield and high diastereoselectivity to deliver (+)-isopulegol, as precedented. ${ }^{26}$ Sulfonylation and aryl transfer proceeded smoothly to yield 1.3 grams of (+)-1 (96\% ee) in $41 \%$ overall yield from commercial materials.

We were curious to see whether radicals derived from prochiral alkenes or linear aryl sulfonates were competent to undergo stereoselective aryl transfer. As shown in Figure 4c, simple linear sulfonate 20a undergoes radical aryl transfer to give $\gamma$-phenyl alcohol $\mathbf{1 9 b}$ in both good yield (73\%) and high stereoselectivity $(16: 1 \mathrm{dr})$. Identical selectivity is observed in reactions involving classically-generated radicals. ${ }^{14 \mathrm{~b}, \mathrm{e}}$ The terminal alkene is key to obtaining high yields because the initial HAT reaction is not regioselective in sterically and electronically-equivalent alkenes, e.g. 1,2-disubstituted alkenes. In a similar manner, the substrate 21a undergoes clean rearrangement with very high stereoselectivity (>20:1) to give the stereochemically-dense alcohol $\mathbf{2 1 b}$ (assigned by x-ray crystallography, see SI). These results suggest such a transformation may find utility beyond the intended application of aryl menthol synthesis.

In summary, we demonstrate an inexpensive, two-step route for the synthesis of arylated menthols from the simple building-blocks isopulegol and arene sulfonyl halides. This strategy leverages HAT to generate quaternary $\mathrm{sp}^{3}-\mathrm{sp}^{2}$ carbon-carbon bonds in exclusive Markovnikov selectivity, and is sufficiently mild to tolerate a wide array of electronically, 
sterically, and functionally differentiated arenes and heteroarenes. More importantly, this HAT strategy for terpene functionalization significantly expands the reach of the monoterpene chiral pool, especially to chiral Lewis bases like pyridine 10, quinoline 13, thiazole $\mathbf{1 6}$ and their derivatives. We anticipate this expansion will prove valuable for the development of new chiral catalysts and reagents.

\section{Supplementary Material}

Refer to Web version on PubMed Central for supplementary material.

\section{Acknowledgments}

Funding Sources

Financial support for this work was provided by the NIH (GM105766 and F31 GM111050 to R.M.) and NSERC (PGS-D3 fellowship to S.C.). Additional support was provided by Eli Lilly, Novartis, Bristol-Myers Squibb, Amgen, Boehringer-Ingelheim, the Sloan Foundation and the Baxter Foundation.

We thank Dr. Milan Gembicky, Dr. Curtis Moore, and Professor Arnold L. Rheingold (University of California, San Diego) for X-ray crystallographic analysis.

\section{References}

1. Breitmaier, E. Terpenes: Flavors, Fragrances, Pharmaca, Pheromones. Wiley VCH; Weinheim: 2006.

2. Liu, W. Handbook of Chiral Chemicals. 2. Ager, D., editor. CRC Press; New York: 2005. p. 59-74.

3. (a) Pronin SV, Shenvi RA. J. Am. Chem. Soc. 2012; 134:19604. [PubMed: 23153381] (b) Pronin SV, Reiher CA, Shenvi RA. Nature. 2013; 501:195. [PubMed: 24025839] (c) Shenvi R, Schnermann MJ. Nat. Prod. Rep. 2015; 32:543. [PubMed: 25514696]

4. (a) Corey EJ, Ensley HE. J. Am. Chem. Soc. 1975; 97:6908. [PubMed: 1184891] (b) Ensley HE, Parnell CA, Corey EJ. J. Org. Chem. 1978; 43:1610.(c) Ort O. Org. Synth. 1987; 65:203. and references therein.

5. Diels-Alder. see ref. 4b; (b) photochemical [2+2]: Nehrings A, Scharf H-D, Runsink J. Angew. Chem. Int. Ed. Engl. 1985; 24:877.(c) glyoxalateene: Whitesell JK, Bhattacharya A, Buchanan CM, Chen HH. Deyo D, James D, Liu C-L, Minton MA. Tetrahedron. 1986; 42:2993.(e) SommeletHauser, see: Tayama E, Kimura H. Angew. Chem. Int. Ed. 2007; 46:8869.

6. Yang D, Ye X-Y, Gu S, Xu M. J. Am. Chem. Soc. 1999; 121:5579.

7. For examples of analogous auxiliaries modified through late-stage functionalization, see: Aoyagi S, Tanaka R, Naruse M, Kibayashi C. J. Org. Chem. 1998; 63:8397.Yanagisawa Y, Yamaguchi H, Nishiyama Y, Morimoto T, Kakiuchi K, Tabata K, Tsutsumi K. Res. Chem. Intermed. 2012; 39:101.Nishiyama Y, Shibata M, Ishii T, Morimoto T, Tanimoto H, Tsutsumi K, Kakiuchi K. Molecules. 2013; 18:1626. [PubMed: 23358321] Saito Y, Segawa Y, Itami K. J. Am. Chem. Soc. 2015; 137:5193. [PubMed: 25860511]

8. Comins DL, Salvador JM. J. Org. Chem. 1993; 58:4656.

9. For examples of analogous auxiliaries synthesized from pulegone, see: Oppolzer W, Kurth M, Reichlin D, Chapuis C, Mohnhaupt M, Moffatt F. Helv. Chim. Acta. 1981; 64:2802.Whitesell JK, Lawrence RM, Chen HH. J. Org. Chem. 1986; 51:4779.D'Angelo J, Maddaluno J. J. Am. Chem. Soc. 1986; 108:8112.Potin D, Dumas F, Maddaluno J. Synth. Commun. 1990; 20:2805.Sato M, Aoyagi S, Yago S, Kibayashi C. Tetrahedron Lett. 1996; 37:9063. Yang D, Xu M, Bian M-Y. Org. Lett. 2001; 3:111. [PubMed: 11429850] Kennedy JWJ, Hall DG. J. Org. Chem. 2004; 69:4412. [PubMed: 15202896] Tsutsumi K, Yanagisawa Y, Furutani A, Morimoto T, Kakiuchi K, Wada T, Mori T, Inoue Y. Chem. Eur. J. 2010; 16:7448. [PubMed: 20461835] Inhülsen I, Akiyama N, Tsutsumi K, Nishiyama Y, Kakiuchi K. Tetrahedron. 2013; 69:782. 
10. According to SciFinder, analogs of $\mathbf{1}$ can be synthesized on demand by Akos, Adlab and Aurora Building Blocks, but we could not find the procedures, yields or costs.

11. For HAT hydrofunctionalization reactions that form $\mathrm{C}-\mathrm{C}$ bonds, see: Isayama $\mathrm{S}$, Mukaiyama T. Chem. Lett. 1989; 18:2005.Wang L-C, Jang H-Y, Roh Y, Lynch V, Schultz AJ, Wang X, Krische MJ. J. Am. Chem. Soc. 2002; 124:9448. [PubMed: 12167039] Gaspar B, Carreira EM. Angew. Chem. Int. Ed. 2007; 46:4519.Gaspar B, Carreira EM. J. Am. Chem. Soc. 2009; 131:13214. [PubMed: 19715273] Lo JC, Yabe Y, Baran PS. J. Am. Chem. Soc. 2014; 136:1304. [PubMed: 24428607] Crossley SWM, Barabé F, Shenvi RA. J. Am. Chem. Soc. 2014; 136:16788. [PubMed: 25398144] Lo JC, Gui J, Yabe Y, Pan C-M, Baran PS. Nature. 2014; 512:343.Dao HT, Li C, Michaudel Q, Maxwell BD, Baran PS. J. Am. Chem. Soc. 2015; 137:8046. [PubMed: 26088401] Zheng J, Wang D, Cui S. Org. Lett. 2015; 17:4572. [PubMed: 26352640] Leggans EK, Barker TJ, Duncan KK, Boger DL. Org. Lett. 2012; 14:1428. [PubMed: 22369097] Choi J, Pulling ME, Smith DM, Norton JR. J. Am. Chem. Soc. 2008; 130:4250. [PubMed: 18335937] Li G, Han A, Pulling ME, Estes DP, Norton JR. J. Am. Chem. Soc. 2012; 134:14662. [PubMed: 22897586]

12. For suggestions that these and similar reactions proceed by M-H HAT, see: Iwasaki K, Wan KK, Oppedisano A, Crossley SWM, Shenvi RA. J. Am. Chem. Soc. 2014; 136:1300. [PubMed: 24428640] King SM, Ma X, Herzon SB. J. Am. Chem. Soc. 2014; 136:6884. [PubMed: 24824195] (c) Ref 7f. For related mechanistic discussion, see: Choi J, Tang L, Norton JR. J. Am. Chem. Soc. 2007; 129:234. [PubMed: 17199304] Ishikawa H, Colby DA, Seto S, Va P, Tam A, Kakei H, Rayl TJ, Hwang I, Boger DL. J. Am. Chem. Soc. 2009; 131:4904. [PubMed: 19292450]

13. Smiles-Truce review: Snape TJ. Chem. Soc. Rev. 2008; 37:2452. [PubMed: 18949118]

14. Related reactions: Lucília M, Mata EN, Motherwell WB, Ujjainwalla F. Tetrahedron Lett. 1997; 38:141.Studer A, Bossart M. Chem. Commun. 1998:2127.Tada M, Uetake T, Hanaoka Y. Chem. Commun. 1999:75.Motherwell WB, Vázquez S. Tetrahedron Lett. 2000; 41:9667.Bossart M, Fässer R, Schoenberger J, Studer A. Eur. J. Org. Chem. 2002:2742.Tada, m, Shijima, H., Nakamura, M. Org. Biomol. Chem. 2003; 1:2499. [PubMed: 12956067] Douglas JJ, Albright H, Sevrin MJ, Cole KP, Stephenson CRJ. Angew. Chem. Int. Ed. 2015; 54:14898.(h) For a review see: Chen Z-M, Zhang X-M, Tu Y-Q. Chem. Soc. Rev. 2015; 44:5220. [PubMed: 25954772]

15. Kalck, P., Serp, P. Green Process Engineering. Poux, M.Cognet, P., Gourdon, C., editors. CRC Press; New York: 2015. p. 425

16. For a review and references to early work, see: Mukaiyama T, Yamada T. Bull. Chem. Soc. Jpn. 1995; 68:17.

17. Recent highlights: Hoffmann RW. Chem. Soc. Rev. 2016; 45:577. [PubMed: 26753913] Simonneau A, Oestreich M. Angew. Chem. Int. Ed. 2015; 54:3556.

18. For transition metal catalyzed radical arylations, see: Gansäuer A, Behlendorf M, von Laufenberg D, Fleckhaus A, Kube C, Sadasivam DV, Flowers RA. Angew. Chem. Int. Ed. 2012; 51:4739.Gansäuer A, Shi L, Otte M, Rosales A, Sancho-Sanz I, Padial NM, Oltra JE. Top. Curr. Chem. 2012; 320:93. [PubMed: 21452081] and references therein.

19. Meerwein arylation shows anti-Markovnikov selectivity with alkenes. For examples, see: Heinrich MR. Chem. Eur. J. 2009; 15:820. [PubMed: 19086045] Hari DP, König B. Angew. Chem. Int. Ed. $2013 ; 52: 4734$.

20. (a) Amrein S, Bossart M, Vasella T, Studer A. J. Org. Chem. 2000; 65:4281. [PubMed: 10891127] (b) Studer A, Bossart M, Steen H. Tetrahedron Lett. 1998; 39:8829.

21. Maneiro M, Fernández B, Gómez-Fórneas E, Rodríguez M, Pedrido R, Romero M, Bermejo M. Z. Anorg. Allg. Chem. 2005; 631:2000.

22. This accelerating effect has been observed previously; see: Isayama S. Bull. Chem. Soc. Jpn. 1990; 63:1305. Waser J, Nambu H, Carreira EM. J. Am. Chem. Soc. 2005; 127:8294. [PubMed: 15941257]

23. Knowles RR, Lin S, Jacobsen EN. J. Am. Chem. Soc. 2010; 132:5030. [PubMed: 20369901]

24. For a review, see: Tauber J, Imbri D, Opatz T. Molecules. 2014; 19:16190. [PubMed: 25310148]

25. (a) Gui J, Pan C-M, Jin Y, Qin T, Lo JC, Lee BJ, Spergel SH, Mertzman ME, Pitts WJ, La Cruz TE, Schmidt MA, Darvatkar N, Natarajan SR, Baran PS. Science. 2015; 348:886. [PubMed: 25999503] (b) Hata E, Kato K, Yamada T, Mukaiyama T. J. Synth. Org. Chem Jpn. 1996; 54:728. 26. Nakatani Y, Kawashima K. Synthesis. 1978:147. 
a. selected examples of chiral induction by $(-)-\mathbf{1}\left[(-)-\mathbf{8}-\mathbf{P M}=\mathbf{R}^{\mathbf{1}}\right]$

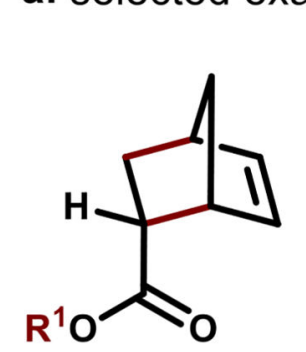

$$
99 \% \text { de }
$$

Diels-Alder<smiles>[R10]C(=O)[C@]1(c2ccccc2)O[C@@H]2OC(C)(C)O[C@H]21</smiles>

$>96 \%$ de

photochemical [2+2]<smiles>[R1]CC=CC[C@@H](O)C(=O)O[Z17]</smiles>

$>99.8 \%$ de

glyoxalate-ene

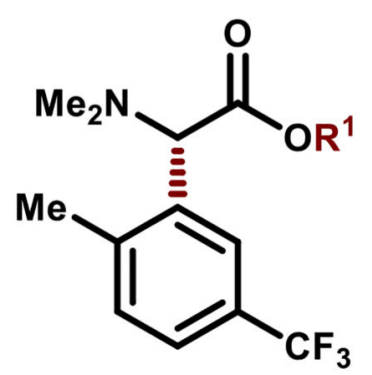

$>96 \%$ de

b. diastereocontrol of (+)-1 en route to (-)-triptonide $\left[(+)-8-\mathbf{P M}=\mathbf{R}^{\mathbf{2}}\right]$<smiles>CCCCC(=O)CCCc1cccc(C(C)C)c1OC</smiles>

2.2 equiv $\mathrm{Mn}(\mathrm{OAc})_{3}$

\section{0 equiv $\mathrm{Yb}(\mathrm{OTf})_{3}$}

$(77 \%, 38: 1 \mathrm{dr})$

[ref 6]

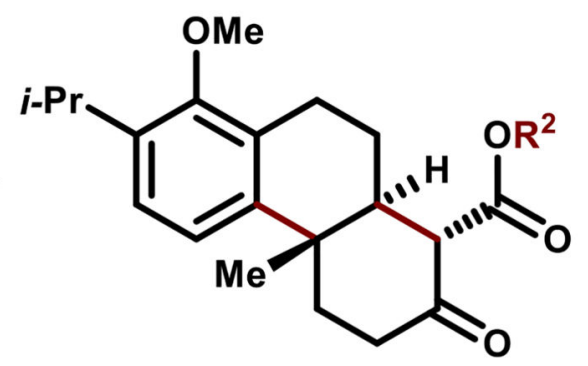

c. HAT enables more direct access to (+)- or (-)-1 and its analogs.<smiles>CC(C)=C1CCC(C)CC1=O</smiles>

$(+)$-pulegone

$3.4 \$ / g(97 \%$ ee $)$

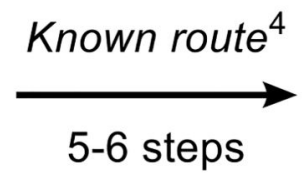

$\mathrm{Ar}=\mathbf{P h}:$

$340 \$ / g(98 \%$ ee $)$
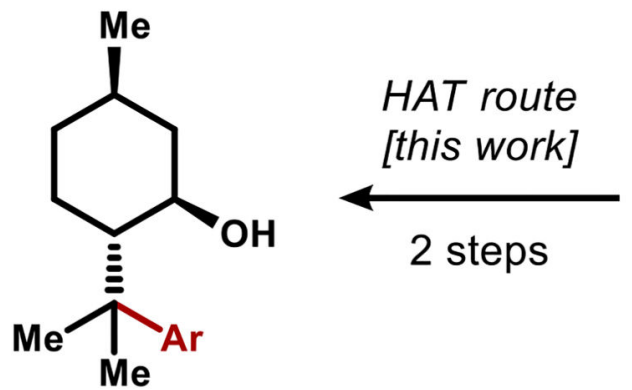

Figure 1.

Uses and synthesis of 8-phenylmenthol (1). 
a. analysis and design

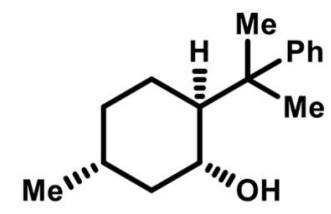

(-)-8-phenylmenthol [(-)-1]<smiles>[X]C(C)C(C)C(C)Br</smiles>

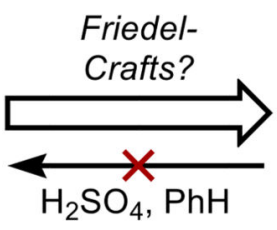

$\mathrm{H}_{2} \mathrm{SO}_{4}, \mathrm{PhH}$<smiles>C=C(C)[C@]1(C)CC[C@@H](C)C[C@H]1O</smiles>

(-)-isopulegol [(-)-2]

1. $\mathrm{PhSO}_{2} \mathrm{Cl}$

2. $[\mathrm{M}-\mathrm{H}]$ HAT

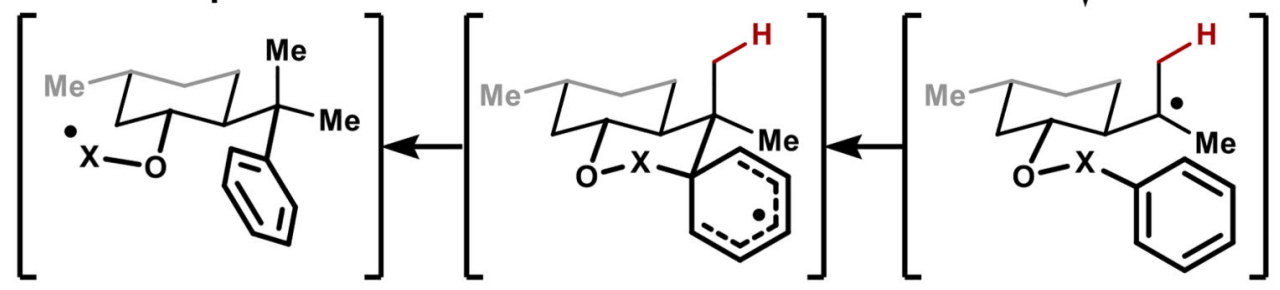

Smiles rearrangement

ipso-attack

radical generation

b. execution<smiles>[R]O[C@@H]1C[C@H](C)CC[C@@H]1C(=C)C</smiles>

A<smiles>C[C@H]1CC[C@@H](C(C)(Br)CBr)[C@H](O)C1</smiles>

$(-)-1$<smiles>[R7][C@H]1C[C@H](C)CC[C@H]1C(C)C[2H]</smiles>

B

\begin{tabular}{|c|c|c|c|}
\hline entry & substrate & conditions & observed products \\
\hline 1 & $\mathbf{R}=\mathbf{B z}$ & $\mathrm{Mn}(\mathrm{dpm})_{3}, \mathrm{PhSiH}_{3}$ & only B \\
\hline 2 & $R=3-C_{3}-B z$ & $\mathrm{Mn}(\mathrm{dpm})_{3}, \mathrm{PhSiH}_{3}$ & only B \\
\hline 3 & $R=2,3,4-F-B z$ & $\mathrm{Mn}(\mathrm{dpm})_{3}, \mathrm{PhSiH}_{3}$ & only B \\
\hline 4 & $R=2,4-F, 3-C_{3}-B z$ & $\mathrm{Mn}(\mathrm{dpm})_{3}, \mathrm{PhSiH}_{3}$ & only B \\
\hline 5 & $\mathbf{R}=\mathrm{SiMe}_{2} \mathbf{P h}$ & $\mathrm{Co}\left(\mathrm{Salen}^{t \mathrm{Bu}, \mathrm{BBu}}\right) \mathrm{Cl}, \mathrm{PhSiH}{ }_{3}, \mathrm{PhH}$ & only recovered $\mathbf{A}$ \\
\hline 6 & $\mathbf{R}=\mathbf{S i P h}_{\mathbf{3}}$ & $\mathrm{Co}\left(\mathrm{Salen}^{t \mathrm{Bu}, \mathrm{tBu}}\right) \mathrm{Cl}, \mathrm{PhSiH}_{3}, \mathrm{PhH}$ & only recovered $\mathbf{A}$ \\
\hline 7 & $\mathrm{R}=\mathrm{SO}_{2} \mathrm{Ph}(3)$ & $\mathrm{Mn}(\mathrm{dpm})_{3}, \mathrm{PhSiH}_{3}$ (see Figure 3) & $(-)-1$ and $B$ \\
\hline
\end{tabular}

Figure 2.

Aryl transfer strategy and execution. 

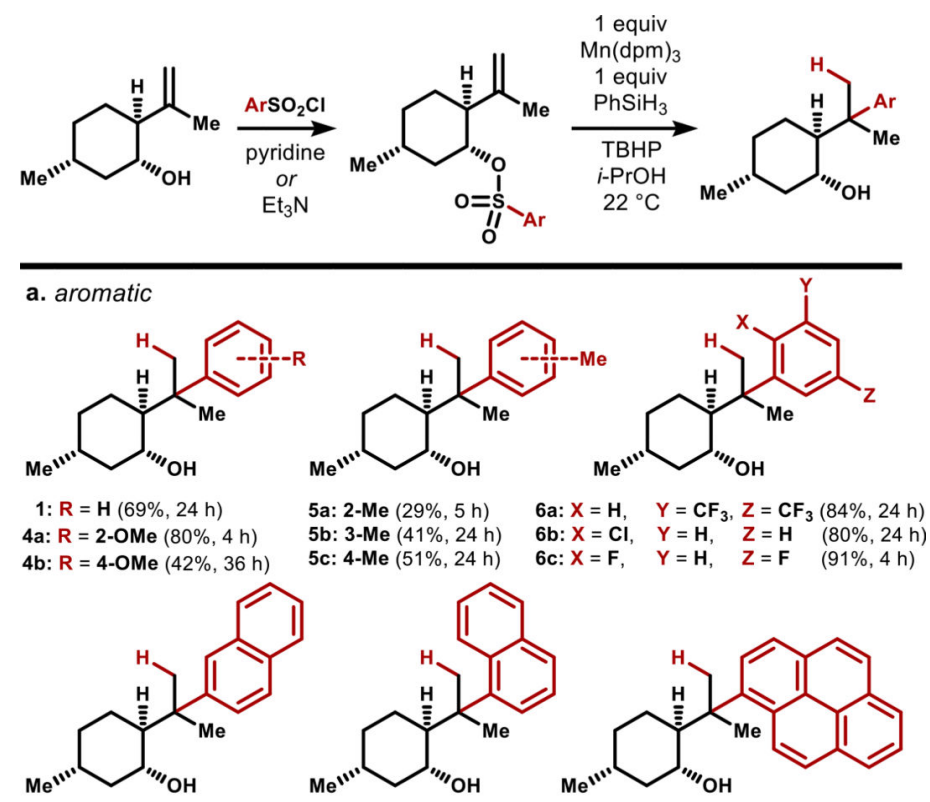

$7(67 \%, 24$ h)

$8(75 \%, 2 \mathrm{~h})$<smiles>C[C@H]1CC[C@@H](Cc2ccc3ccc4cccc5ccc2c3c45)[C@H](O)C1</smiles>

b. heteroaromatic

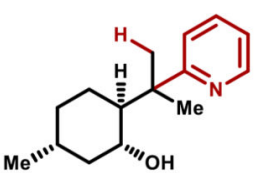

$10(89 \%, 24 \mathrm{~h})$<smiles>C[C@H]1CC[C@H](C(C)(C)c2cccc3cccnc23)[C@H](O)C1</smiles>

$13(78 \%, 24 \mathrm{~h})$

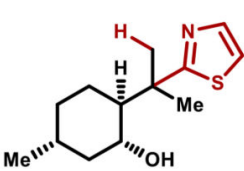

$16(71 \%, 23 \mathrm{~h})$

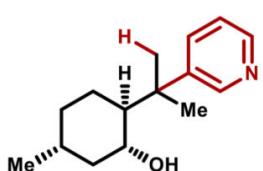

$11(82 \%, 48$ h)

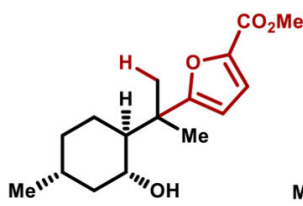

$14(30 \%, 22 \mathrm{~h})$

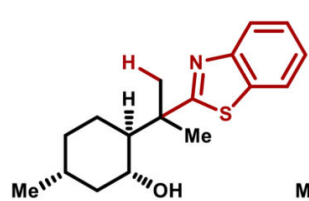

$17(57 \%, 3 \mathrm{~h})$

$9(24 \%, 24 \mathrm{~h})$
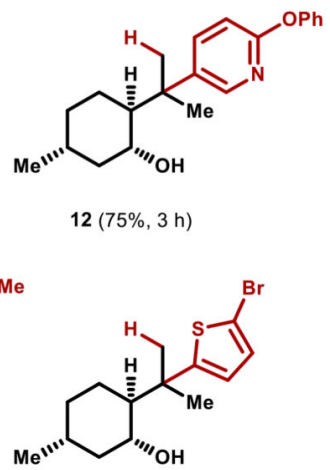

$15(78 \%, 23 \mathrm{~h})$

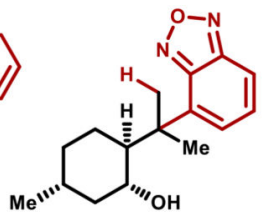

$18(15 \%, 24 \mathrm{~h})$

Figure 3.

Survey of aryl sulfonate transfer using (-)-isopulegol. 
a. Multigram synthesis of (-)-1 using $\mathrm{Mn}(\mathrm{OAc})_{3}$<smiles>C=C(C)[C@H]1CC[C@@H](C)C[C@H]1OS(=O)(=O)c1ccccc1</smiles>

$3(15 g)$
$5 \mathrm{~mol} \%$

$\mathrm{Mn}(\mathrm{dpm})_{3}$

1 equiv $\mathrm{PhSiH}_{3}$

1 equiv $\mathrm{Mn}(\mathrm{OAc})_{3}$

2 equiv TBHP

i- $\mathrm{PrOH}(0.05 \mathrm{M})$

$14 \mathrm{~d}, 4{ }^{\circ} \mathrm{C}(78 \%)$<smiles>C[C@H]1CC[C@H](C(C)(C)c2ccccc2)[C@H](O)C1</smiles>

$(-)-1[9.2 g]$

b. Facile synthesis of (+)-1 from (-)-citronellal.<smiles>CC(C)=CCC[C@@H](C)CC=O</smiles>

(S)-(-)-citronellal (19)
1. $\mathrm{ZnBr}_{2}, \mathrm{CH}_{2} \mathrm{Cl}_{2}$ $(68 \%)[70 \%$ lit.]

2. $\mathrm{PhSO}_{2} \mathrm{Cl}$, py $(88 \%)$

3. $\mathrm{Mn}(\mathrm{OAc})_{3}$ $7.5 \mathrm{~mol} \% \mathrm{Mn}(\mathrm{dpm})_{3}$ i-PrOH (68\%)

(41\% overall)

c. Selected examples of remote stereoinduction<smiles>C=CCC(OS(=O)(=O)c1ccccc1)C(C)C</smiles>

$( \pm)-20 a$

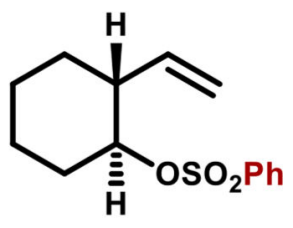

(士)-21a
1 equiv $\mathrm{Mn}(\mathrm{dpm})_{3}$ 1 equiv $\mathrm{PhSiH}_{3}$

i-PrOH, $22{ }^{\circ} \mathrm{C}, 5 \mathrm{~h}$ $(73 \%, 16: 1 \mathrm{dr})$

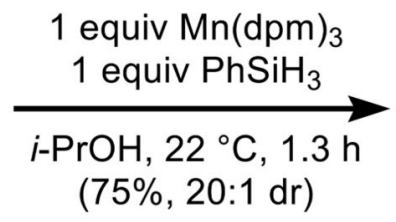

[see SI for x-ray]<smiles>C[C@H]1CC[C@H](C(C)(C)c2ccccc2)[C@H](O)C1</smiles>

$(+)-1[1.3 g]$<smiles>[CH][C@@H](C[C@H](O)C(C)C)c1ccccc1</smiles>

( \pm )-20b<smiles></smiles>

(士)-21b

Figure 4.

Further applications for HAT-initiated aryl transfer. 